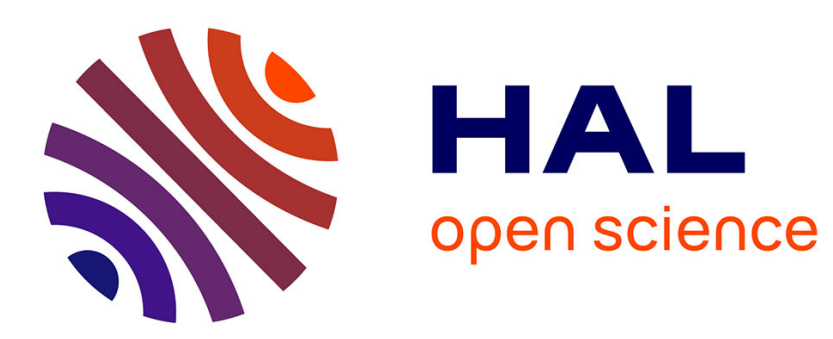

\title{
On the robust stability of some parameter-dependent linear systems Solutions via matrix pencil techniques
}

Jie Chen, Peilin Fu, Silviu-Iulian Niculescu

\section{To cite this version:}

Jie Chen, Peilin Fu, Silviu-Iulian Niculescu. On the robust stability of some parameter-dependent linear systems Solutions via matrix pencil techniques. 15th Mediterranean Conference on Control and Automation, Jun 2007, Athens, Greece. 10.1109/MED.2007.4433795 . hal-02194458

\section{HAL Id: hal-02194458 \\ https://hal.science/hal-02194458}

Submitted on 26 Jul 2019

HAL is a multi-disciplinary open access archive for the deposit and dissemination of scientific research documents, whether they are published or not. The documents may come from teaching and research institutions in France or abroad, or from public or private research centers.
L'archive ouverte pluridisciplinaire HAL, est destinée au dépôt et à la diffusion de documents scientifiques de niveau recherche, publiés ou non, émanant des établissements d'enseignement et de recherche français ou étrangers, des laboratoires publics ou privés. 


\title{
On the Robust Stability of Some Parameter-Dependent Linear Systems: Solutions via Matrix Pencil Techniques
}

\author{
Jie Chen, Peilin Fu, and Silviu-Iulian Niculescu
}

\begin{abstract}
This note focuses on deriving stability conditions for a class of linear parameter-dependent systems in a statespace representation. More precisely, we will compute the set of parameters for which the characteristic roots are located on the imaginary axis, and next we will give the characterization of the way such critical roots are crossing the imaginary axis. The methodology considered makes use of the computation of the generalized eigenvalues of an appropriate matrix pencil combined with an operator perturbation approach for deriving the crossing direction. Finally, the particular case of parameterdependent polynomials will be also considered, and the stability analysis of time-delay systems is also revisited in this perspective.
\end{abstract}

\section{INTRODUCTION}

The aim of the paper is to discuss in extent the asymptotic behavior of imaginary eigenvalues of some matrix polynomials. The main interest of this discussion lies in deriving less conservative methods for dynamical systems which are marginally stable. We adopt an operator perturbation approach (see, e.g., [9], [8]) to the corresponding switch problem. Indeed, the approach seeks to recast the zero asymptotic analysis problem as one of eigenvalue perturbation. This enables us to obtain readily computable results that fully characterize the variation properties of the critical zeros at the critical roots of the corresponding system. A special attention will be paid to the cases when the critical characteristic root on the imaginary axis is not simple. Thus, both semi-simple and multiple, but not semi-simple eigenvalue cases will be largely discussed, and explicit formula of the corresponding crossing directions evaluation will be derived.

As a byproduct of the analysis proposed in the note several classical problems are revised and discussed in this new perspective. In particular, we will emphasize the connections with the Routh-Hurwitz criterion and root locus method. Finally, connections to delay systems, and in particular the so-called pseudo-delay technique are also considered.

The paper is organized as follows: Section II includes the Problem formulation. Some preliminary results are briefly outlined in Section III. The main results are presented in both state-space representation and parameter-dependent polynomial cases in Section IV, and V respectively. Various

This research is supported in part by CNRS/France and NSF/USA

J. Chen is with the Department of Electrical Engineering, University of California, Riverside, CA 92521, USA. jchendee. ucr. edu

$\mathrm{P} . \mathrm{Fu}$ is with the Department of Electrical Engineering, University of California, Riverside, CA 92521, USA. pfu@ee.ucr.edu

S.-I. Niculescu is with the Laboratoire de Signaux et Systèmes (L2S), Supélec, 3, rue Joliot Curie, 91192, Gif-sur-Yvette, France. Silviu.Niculesculls.s.supelec.fr comments and useful observations in the context of timedelay systems are given in Section VI. Some comments and remarks end the paper.

The following notations will be used throughout the paper. Let $\mathbb{R}$ be the set of real numbers, $\mathbb{C}$ the set of complex numbers, and $\mathbb{R}_{+}$the set of nonnegative real numbers. Denote the open right half plane by $\mathbb{C}_{+}:=\{s: \Re(s)>0\}$, the closed right half plane by $\overline{\mathbb{C}}_{+}$, and the imaginary axis by $\partial \mathbb{C}_{+}$. Similarly, denote the open unit disc by $\mathbb{D}$, the unit circle by $\partial \mathbb{D}$, and the closed exterior of the unit disc by $\mathbb{D}^{c}$. For a matrix $A$, denote its spectrum by $\sigma(A)$, and the ith eigenvalue by $\lambda_{i}(A)$. For a matrix pair $(A, B)$, denote the set of all its generalized eigenvalues by $\sigma(A, B)$, i.e.,

$$
\sigma(A, B):=\{\lambda \in \mathbb{C}: \operatorname{det}(A-\lambda B)=0\} .
$$

\section{PROBlem Formulation}

Consider the following class of dynamical systems:

$$
\dot{x}(t)=A(p) x(t),
$$

where $x \in \mathbb{R}^{n}$, and the mapping $A:\left[p_{\text {min }}, p_{\max }\right] \mapsto \mathbb{R}^{n \times n}$ is given by the matrix polynomial:

$$
A(p):=\sum_{k=0}^{q} A_{k} p^{k} .
$$

In the sequel, a parameter-dependent system defined by (1)(2) is called marginally stable for some parameter $p=p_{c}$ if all the eigenvalues of the corresponding characteristic equation are located in $\mathbb{C}_{-}$or on the imaginary axis $\partial \mathbb{C}_{+}$ (not any strictly unstable roots).

The problem considered in this paper is to give a complete characterization of the stability of (1)-(2) with respect to the parameter $p$. We shall exploit the particular structure of the parameter-dependence in order to compute all the parameter-crossing values for which a change in the stability may take place. Furthermore, the way the eigenvalues are crossing the imaginary axis will be explicitly derived. Define now the set $\mathcal{P}$ as the set of all parameters for which the original system is marginally stable. Such a set will be called the parameter-crossing set. As discussed in the sequel, the computation of $\mathcal{P}$ is reduced to the computation of the generalized eigenvalue of some appropriate matrix pencil of finite dimension, and a simple matrix eigenvalue test. All the cases (simple, multiple semi-simple, and not semi-simple eigenvalues) will be discussed in some extent. Various results from robust stability and robust control will be revisited in this formalism. 


\section{Preliminary Results}

In this section, we introduce the operator perturbation theory for matrix eigenvalue problems, which concerns how the eigenvalues of a matrix function may vary with respect to a small perturbation. The development is based on the classical treatise of Kato [9], but goes further beyond.

\section{A. First-order analysis}

Consider a matrix operator $T(x)$ of a real variable $x$. Suppose that in the neighborhood of $x=0$, the perturbed operator $T(x)$ is holomorphic, or equivalently, can be expanded into the power series,

$$
T(x)=T(0)+x T^{\prime}(0)+x^{2} T^{\prime \prime}(0)+\cdots .
$$

Let $D_{0}$ be a small disk near $x=0$ but excluding $x=0$. It is known that for $x \in D_{0}$, any semi-simple eigenvalue of $T(x)$, namely the semi-simple root of the characteristic equation

$$
\operatorname{det}(T(x)-\xi I)=0,
$$

is an analytic functions of $x$ and can also be expressed as a power series in $x$; here by a semi-simple eigenvalue, we mean a repeated but diagonalizable eigenvalue. This fact is summarized in Lemma 1.

Lemma 1: [9] Let $\lambda^{(0)}$ be a semi-simple eigenvalue of $T(0)$ with multiplicity $m$, and $P$ be the eigenprojection for $\lambda^{(0)}$, that is,

$$
P=\frac{1}{2 \pi j} \oint_{\Gamma}(\xi I-T(0))^{-1} d \xi
$$

where $\Gamma$ is a positively-oriented closed contour enclosing $\lambda^{(0)}$ but no other eigenvalues of $T(0)$. Then the corresponding eigenvalues of $T(x)$ are analytic in $x$ and have the form

$$
\mu_{i}(x)=\lambda^{(0)}+\lambda_{i}^{(1)} x+o\left(x^{2}\right), \quad i=1, \ldots, m,
$$

where $\lambda_{i}^{(1)}$ are the eigenvalues of $P T^{\prime}(0) P$.

With no loss of generality, let $\lambda^{(0)}$ be ordered as the first eigenvalue of $T(0)$ with multiplicity $m$. Then $T(0)$ can be decomposed as

$$
T(0)=Q \Sigma R=\left[\begin{array}{ll}
Q_{1} & Q_{2}
\end{array}\right]\left[\begin{array}{cc}
\Sigma_{1} & 0 \\
0 & \Sigma_{2}
\end{array}\right]\left[\begin{array}{l}
R_{1} \\
R_{2}
\end{array}\right],
$$

where $\Sigma_{1}$ is diagonal with diagonal entries as $\lambda^{(0)}, R=$ $Q^{-1}=\left[\begin{array}{lll}r_{1}^{T} & \cdots & r_{n}^{T}\end{array}\right]^{T}$, and $Q=\left[\begin{array}{lll}q_{1} & \cdots & q_{n}\end{array}\right]$ consist of the eigenvectors and generalized eigenvectors of of $T(0)$. The following lemma shows how $\lambda_{i}^{(1)}$ may be computed.

Lemma 2: [4] Let $T(0)$ be partitioned as in (7). Then $\lambda_{i}^{(1)}$, $i=1, \ldots, m$, in (6) are the eigenvalues of $R_{1} T^{\prime}(0) Q_{1}$.

Consider next the case that $\lambda^{(0)}$ is not a semi-simple but repeated eigenvalue of $T(0)$ with multiplicity $\mathrm{m}$. In this case, $T(0)$ admits a Jordan decomposition in which $\Sigma$ is block diagonal with diagonal Jordan blocks, and $Q_{1}$ consists of the generalized eigenvectors associated with $\lambda^{(0)}$. In particular,

$$
\Sigma_{1}=\left[\begin{array}{cccc}
\lambda^{(0)} & 1 & \cdots & 0 \\
0 & \ddots & \ddots & 0 \\
\vdots & & \ddots & 1 \\
0 & \cdots & \cdots & \lambda^{(0)}
\end{array}\right]
$$

The eigenvalue of $T(x)$ can no longer be expanded in the form of (6), but instead as a Puiseux series:

Lemma 3: [4] Let $\lambda^{(0)}$ be a non-semi-simple eigenvalue of $T(0)$ with multiplicity $m$. Then the corresponding eigenvalues of $T(x)$ have the form

$$
\mu_{i}(x)=\lambda^{(0)}+\left(\gamma_{i}^{(1)}\right)^{\frac{1}{m}} x^{\frac{1}{m}}+\cdots, \quad i=1, \ldots, m,
$$

where $\gamma_{i}^{(1)}=r_{m} T^{\prime}(0) q_{1}$.

\section{B. Second-Order Asymptotic Expansion}

The first-order asymptotic series introduced in the preceding subsection can be further developed to include terms of higher orders. In this section we present formulas for computing the coefficients of the asymptotic series up to the second-order as presented in [6] by using results by Kato [9].

Define the operator-valued function

$$
\Upsilon(\xi)=(T(0)-\xi I)^{-1},
$$

which is known as the resolvent of $T(0)$. It is obvious that the singularities of $\Upsilon(\xi)$ are the eigenvalues of $T(0)$. Let $\lambda^{(0)}$ be a semi-simple eigenvalue of $T(0)$. Then $\Upsilon(\xi)$ can be expanded as a Laurent series at $\xi=\lambda^{(0)}$, that is,

$$
\begin{aligned}
\Upsilon(\xi) & =-\left(\xi-\lambda^{(0)}\right)^{-1} P-\sum_{n=1}^{\infty}\left(\xi-\lambda^{(0)}\right)^{-n-1} D_{n} \\
& +\sum_{n=0}^{\infty}\left(\xi-\lambda^{(0)}\right)^{n} S_{n+1},
\end{aligned}
$$

where $P, D_{n}$ and $S_{n+1}$ are corresponding coefficient matrices. Evidently, the matrix $P$, known as the eigenprojection for $\lambda^{(0)}$, can be found as

$$
P=-\frac{1}{2 \pi j} \oint_{\Gamma} \Upsilon(\xi) d \xi=\frac{1}{2 \pi j} \oint_{\Gamma}(\xi I-T(0))^{-1} d \xi,
$$

where $\Gamma$ is a positively-oriented closed contour enclosing $\lambda^{(0)}$ but no other eigenvalues of $T(0)$. The holomorphic part in the Laurent expansion is called the reduced resolvent of $T(0)$ with respect to the eigenvalue $\lambda^{(0)}$, denoted as

$$
S(\xi)=\sum_{n=0}^{\infty}\left(\xi-\lambda^{(0)}\right)^{n} S_{n+1} .
$$

Let $S=S\left(\lambda^{(0)}\right)$, namely the value of the reduced resolvent of $T(0)$ at $\xi=\lambda^{(0)}$. Then it is obvious that $S=S_{1}$.

Lemma 4: [6] For any matrix $T(0)$ decomposed in the form of (7), where $\Sigma_{1}$ is in Jordan form with diagonal entries as $\lambda^{(0)}$, the reduced resolvent at $\xi=\lambda^{(0)}$ is equal to

$S=Q\left[\begin{array}{cc}0 & 0 \\ 0 & \left(\Sigma_{2}-\lambda^{(0)} I\right)^{-1}\end{array}\right] R=Q_{2}\left(\Sigma_{2}-\lambda^{(0)} I\right)^{-1} R_{2}$.

The following Lemma given in [9] provides the result on the second order perturbation of $T(x)$ when all the eigenvalues of $T(0)$ are semi-simple.

Lemma 5: [9] Let $\lambda^{(0)}$ be a semi-simple eigenvalue of $T(0), \lambda_{i}^{(1)}$ be a semi-simple eigenvalue of $P T^{\prime}(0) P$ with the eigen-projection $P_{i}^{(1)}$, that is

$$
P_{i}^{(1)}=\oint_{\Gamma_{i}}\left(\xi I-P T^{\prime}(0) P\right)^{-1} d \xi,
$$


where $\Gamma_{i}$ is a positively-oriented closed contour enclosing $\lambda_{i}^{(1)}$ but no other eigenvalues of $P T^{\prime}(0) P$. Then $T(x)$ has $d=\operatorname{dim} P_{i}^{(1)}$ repeated eigenvalues of the form

$$
\mu_{i p}(x)=\lambda^{(0)}+x \lambda_{i}^{(1)}+x^{2} \mu_{i p}^{(2)}+o\left(x^{2}\right), \quad p=1, \cdots, d,
$$

where $\mu_{i p}^{(2)}$ are the repeated eigenvalues of $P_{i}^{(1)} T^{(2)} P_{i}^{(1)}$ with $T^{(2)}=T^{\prime \prime}(0)-T^{\prime}(0) S T^{\prime}(0)$, and

$$
P_{i}^{(1)} T^{(2)} P_{i}^{(1)}=P_{i}^{(1)} T^{\prime \prime}(0) P_{i}^{(1)}-P_{i}^{(1)} T^{\prime}(0) S T^{\prime}(0) P_{i}^{(1)} .
$$

The eigenvalues of $P_{i}^{(1)} T^{(2)} P_{i}^{(1)}$ can be computed in a manner similar to that in the first-order analysis.

Lemma 6: [6] Let $\lambda^{(0)}$ be a semi-simple eigenvalue of $T(0), \lambda_{i}^{(1)}$ be a semi-simple eigenvalue of $P T^{\prime}(0) P$. Let also $T(0)$ be decomposed as in (7), and $R_{1} T^{\prime}(0) Q_{1}$ be decomposed as

$$
\begin{aligned}
R_{1} T^{\prime}(0) Q_{1} \\
\quad=Q^{(2)} \Sigma^{(2)} R^{(2)} \\
\quad=\left[\begin{array}{ll}
Q_{1}^{(2)} & Q_{2}^{(2)}
\end{array}\right]\left[\begin{array}{cc}
\Sigma_{1}^{(2)} & 0 \\
0 & \Sigma_{2}^{(2)}
\end{array}\right]\left[\begin{array}{l}
R_{1}^{(2)} \\
R_{2}^{(2)}
\end{array}\right],
\end{aligned}
$$

where $\Sigma_{1}^{(2)}$ is the Jordan block corresponding to the eigenvalue $\lambda_{i}^{(1)}$. Then the eigenvalues of $P_{i}^{(1)} T^{(2)} P_{i}^{(1)}$ are those of the matrix $R^{(2)} R_{1} T^{(2)} Q_{1} Q_{1}^{(2)}$.

\section{MAIN RESULTS}

Introduce now the following matrix pencil $\Lambda: \mathbb{C} \mapsto$ $\mathbb{C}^{q n^{2} \times q n^{2}}, \Lambda(\lambda):=\lambda U+V$, where the matrices $U$, and $V$ are given by:

$$
\begin{aligned}
U= & {\left[\begin{array}{ccccc}
I_{n^{2}} & 0 & 0 & \ldots & 0 \\
0 & I_{n^{2}} & 0 & \ldots & 0 \\
\vdots & & & \ddots & \vdots \\
0 & 0 & 0 & \ldots & B_{q}
\end{array}\right] } \\
V= & {\left[\begin{array}{ccccc}
0 & -I_{n^{2}} & 0 & \ldots & 0 \\
0 & 0 & -I_{n^{2}} & \ldots & 0 \\
\vdots & & & \ddots & \vdots \\
B_{0} & B_{1} & B_{2} & \ldots & B_{q-1}
\end{array}\right], }
\end{aligned}
$$

where $B_{k}:=A_{k} \oplus A_{k}^{T}$, for all $k=\overline{0, q}$.

\section{A. Stability crossing set}

With the definitions, and the notations above, we have:

Lemma 7: Consider the system (1)-(2) for some $p_{0} \in$ $\left[p_{\min }, p_{\max }\right]$. Then $p_{0}$ is a parameter-crossing value if and only if the following conditions are satisfied simultaneously:

(i) $p_{0} \in \sigma(U, V)$, that is $p_{0}$ is a generalized eigenvalue of the matrix pencil $\Lambda$, and

(ii) $\sigma\left(A\left(p_{0}\right)\right) \cap j \mathbb{R} \neq \varnothing$, that is the real matrix $A\left(p_{0}\right)$ has at least one eigenvalue on the imaginary axis.

Remark 1 (Symmetric eigenvalues): The condition (i) in the Lemma 7 is not sufficient for crossing existence. Indeed, by construction, the spectrum of $\Lambda$ includes all the eigenvalues of the matrix $A(p)$ which are symmetric with respect to the origin, and thus we need to exclude the symmetric roots which are not located on $\partial \mathbb{C}_{+}$.
Since, excepting $\mathcal{P}$, there are no other crossings with respect to $\partial \mathbb{C}_{+}$, let $\operatorname{card}(\mathcal{P})=\ell$, and let $p_{1}, p_{2}, \ldots, p_{\ell}$ be corresponding ordered crossing parameters. The continuity properties of the characteristic roots with respect to the parameters [8] allows concluding that for all $p \in\left(p_{k}, p_{k+1}\right)$, $k=1, \ldots \ell-1$, the number of strictly unstable characteristic roots (i.e. the instability degree) of (1)-(2) is constant. In conclusion, the characterization of the parameter-crossing set $\mathcal{P}$ is complete.

\section{B. Crossing direction characterization}

1) Simple crossing characteristic roots: Consider now that the root $s=j \omega_{0}$ corresponding to some parameter $p_{0} \in \mathcal{P}$ is simple. Let $u_{0}\left(v_{0}^{*}\right)$ be the corresponding right(left-) eigenvectors, that is:

$$
\left\{\begin{array}{l}
\left(j \omega_{0} I_{n}-A\left(p_{0}\right)\right) u_{0}=0 \\
v_{0}^{*}\left(j \omega_{0} I_{n}-A\left(p_{0}\right)\right)=0 .
\end{array}\right.
$$

Without any loss of generality, we can assume that the rightand left-eigenvectors $u_{0}, v_{0}^{*}$ satisfy the regularity condition $v_{0}^{*} u_{0}=1$. Then:

Proposition 1: Let $j \omega_{0}$ be a simple eigenvalue of $A\left(p_{0}\right)$. Thus, for $p$ sufficiently close to $p_{0}$ but $p>p_{0}$, there exist a characteristic zero entering the right-half plane (or vice versa) if the following condition is satisfied:

$$
\operatorname{Re}\left\{v_{0}^{*}\left(\sum_{k=1}^{q} k A_{k} p_{0}^{k-1}\right) u_{0}\right\}<0(>0) .
$$

Additionally, if

$$
\operatorname{Re}\left\{v_{0}^{*}\left(\sum_{k=1}^{q} k A_{k} p_{0}^{k-1}\right) u_{0}\right\}=0,
$$

then for any $p$ sufficiently close to $p_{0}$ but $p>p_{0}, j \omega_{0}$ enters the right-half plane (or vice versa) if

$$
\operatorname{Re}\left\{v_{0}^{*}\left(\sum_{k=2}^{q} k(k-1) A_{k} p_{0}^{k-2}\right) u_{0}\right\}<0 \quad(>0) \text {, }
$$

In other words, the crossing direction for simple roots is given by the sign of (16) easy to evaluate since $u_{0}, v_{0}^{*}, A_{k}$, $k=1, \ldots, m$ and $p_{0}=j \omega_{0}$ are known. It is important to point out that such a quantity is non-zero since the corresponding characteristic root is simple, but, as mentioned in Proposition 1, it can be on the imaginary axis.

2) Semi-simple crossing characteristic roots: Consider now the case of a multiple, but semi-simple eigenvalue $j \omega_{0}$ on the imaginary axis. Using the arguments proposed in the previous section, simple computations lead to the following:

Proposition 2: Let $j \omega_{0}$ be a semi-simple eigenvalue of $A\left(p_{0}\right)$ with multiplicity $m$ and $\lambda_{i}^{(1)}$ be a semi-simple eigenvalue of

$$
-R_{1}\left(\sum_{k=1}^{q} k A_{k} p_{0}^{k-1}\right) Q_{1}
$$

with multiplicity $d$. Then for any $p$ sufficiently close to $p_{0}$, the characteristic zeros corresponding to $j \omega_{0}$ can be expanded by the power series

$$
j \omega_{0}+\lambda_{i}^{(1)}\left(p-p_{0}\right)+\mu_{i l}^{(2)}\left(p-p_{0}\right)^{2}+o\left(\left(p-p_{0}\right)^{3}\right),
$$


with

$$
\begin{aligned}
\lambda_{i}^{(1)} & =-\lambda_{i}\left[R_{1}\left(\sum_{k=1}^{q} k A_{k} p_{0}^{k-1}\right) Q_{1}\right], \\
i=1,2, \cdots, m, & \\
\mu_{i l}^{(2)}=-\lambda_{l}\left[R_{1}^{(2)} R_{1}\left(\sum_{k=2}^{q} k(k-1) A_{k} p_{0}^{k-2}\right) Q_{1} Q_{1}^{(2)}\right], & l=1,2, \cdots, d,
\end{aligned}
$$

where $Q_{1}^{(2)}=\left[\begin{array}{llll}\tilde{q}_{1} & \tilde{q}_{2} & \cdots & \tilde{q}_{d}\end{array}\right]$ and $R_{1}^{(2)}=\left[\begin{array}{llll}\tilde{r}_{1}^{T} & \tilde{r}_{2}^{T} & \cdots & \tilde{r}_{d}^{T}\end{array}\right]^{T}$, with $\tilde{q}_{p}$ and $\tilde{r}_{p}$ the right and left eigenvectors of

$$
-R_{1}\left(\sum_{k=1}^{q} k A_{k} p_{0}^{k-1}\right) Q_{1}
$$

associated with $\lambda_{i}^{(1)}$.

(i) For $p$ sufficiently close to $p_{0}$ but $p>p_{0}$, there are at least $h(h \leq m)$ of characteristic zeros entering the right-half plane (or vice versa) if $h$ of the eigenvalues satisfy the condition

$$
\begin{aligned}
& \operatorname{Re}\left\{\lambda_{i}\left[R_{1}\left(\sum_{k=1}^{q} k A_{k} p_{0}^{k-1}\right) Q_{1}\right]\right\}<0(>0), \\
& i=1,2, \cdots, m .
\end{aligned}
$$

(ii) If

$$
\operatorname{Re}\left\{\lambda_{i}\left[R_{1}\left(\sum_{k=1}^{q} k A_{k} p_{0}^{k-1}\right) Q_{1}\right]\right\}=0,
$$

then for $p$ sufficiently close to $p_{0}$ but $p>p_{0}$, there are at least $h^{\prime}\left(h^{\prime} \leq d\right)$ of characteristic zeros entering the right-half plane (or vice versa) if $h^{\prime}$ of the eigenvalues $\mu_{i l}^{(2)}$ satisfy the condition

$$
\begin{array}{r}
\operatorname{Re}\left\{\lambda_{l}\left[R_{1}^{(2)} R_{1}\left(\sum_{k=2}^{q} k(k-1) A_{k} p_{0}^{k-2}\right) Q_{1} Q_{1}^{(2)}\right]\right\} \\
<0(>0), l=1,2, \cdots, d .(20)
\end{array}
$$

Finally, the last case of interest in this situation is given by the case when the eigenvalues $\lambda_{i}^{(1)}$ in Proposition 2 are not semi-simple:

Proposition 3: Let $j \omega_{0}$ be a semi-simple eigenvalue of $A\left(p_{0}\right)$ with multiplicity $m$ and $\lambda_{i}^{(1)}$ be a repeated eigenvalue of

$$
-R_{1}\left(\sum_{k=1}^{q} k A_{k} p_{0}^{k-1}\right) Q_{1}
$$

with multiplicity $d$. Suppose that $\lambda_{i}^{(1)}$ is not semi-simple.

Then for any $p$ sufficiently close to $p_{0}$ but $p>p_{0}$, the characteristic zeros corresponding to $j \omega_{0}$ can be expanded by the Puiseux series

$$
\begin{aligned}
j \omega_{0} & +\lambda_{i}^{(1)}\left(p-p_{0}\right)+\left|\tilde{r}_{d} \tilde{T} \tilde{q}_{1}\right|^{\frac{1}{d}} e^{j \frac{2 h \pi+\pi+\theta}{d}}\left(p-p_{0}\right)^{1+\frac{1}{d}} \\
& +\cdots, \quad h=0,1, \cdots, d-1
\end{aligned}
$$

where

$$
\tilde{T}=R_{1}^{(2)} R_{1}\left(\sum_{k=2}^{q} k(k-1) A_{k} p_{0}^{k-2}\right) Q_{1} Q_{1}^{(2)},
$$

where $\theta \in[0,2 \pi]$ is the phase angle of $\tilde{r}_{d} \tilde{T} \tilde{q}_{1}$, and $\tilde{q}_{1}$ and $\tilde{r}_{d}$ are obtained from the Jordan decomposition

$$
Q_{1} R_{1}\left(-\sum_{k=1}^{q} k A_{k} p_{0}^{k-1}\right) Q_{1} R_{1}=\tilde{Q} \tilde{\Sigma} \tilde{R},
$$

with $\tilde{Q}=\left[\begin{array}{lllll}\tilde{q}_{1} & \cdots & \tilde{q}_{d} & \cdots & \tilde{q}_{m}\end{array}\right]$ and $\tilde{R}=\tilde{Q}^{-1}=$ $\left[\begin{array}{lllll}\tilde{r}_{1}^{T} & \cdots & \tilde{r}_{d}^{T} & \cdots & \tilde{r}_{m}^{T}\end{array}\right]^{T}$.

Hence, for $p$ sufficiently close to $p_{0}$ but $p>p_{0}$, the number of critical zeros entering the right-half plane (or vice versa) can be determined by the condition

$$
\cos \left(\frac{2 h \pi+\pi+\theta}{d}\right)>0 \quad(<0), \quad h=0, \cdots, d-1 .
$$

Propositions 2 and 3 reveal a fundamental difference between the asymptotic behaviors of a semi-simple eigenvalue and one that is not. Take for instance Proposition 3, for a repeated eigenvalue that is not semi-simple, the result shows that, precluding the case that

$$
r_{m}\left(\sum_{k=1}^{q} k A_{k} p_{0}^{k-1}\right) q_{1}=0,
$$

whether the zero will enter the right-half plane is solely determined by the multiplicity of the zero and the phase angle $\theta$. In this case, the branches of the zero will almost generically enter the right-half plane. Note that in the degenerate case $m=1$, Proposition 3 also reduces to Proposition 2 .

3) Multiple non semi-simple characteristic roots: Finally, consider the case of a multiple, but not semi-simple eigenvalue $j \omega_{0}$ on the imaginary axis. Then:

Proposition 4: Let $j \omega^{*}$ be a repeated eigenvalue of $\sum_{k=0}^{q} A_{k} e^{-j \omega^{*} k \tau^{*}}$ with multiplicity $m$. Suppose that $j \omega_{0}$ is not semi-simple.

Then for any $p$ sufficiently close to $p_{0}$ but $p>p_{0}$, the characteristic zeros corresponding to $p_{0}$ can be expanded by the Puiseux series

$$
\begin{aligned}
j \omega_{0} & +\left|r_{m}\left(\sum_{k=1}^{q} k A_{k} p_{0}^{k-1}\right) q_{1}\right|^{\frac{1}{m}} e^{j \frac{2 h \pi+\pi+\theta}{m}} \\
& \times\left(p-p_{0}\right)^{\frac{1}{m}}+\cdots, \quad h=0,1, \cdots, m-1,
\end{aligned}
$$

where $\theta \in[0,2 \pi]$ is the phase angle of

$$
r_{m}\left(\sum_{k=1}^{q} k A_{k} p_{0}^{k-1}\right) q_{1}
$$

and $q_{1}$ and $r_{m}$ are obtained from the Jordan decomposition

$$
\sum_{k=0}^{q} A_{k} p_{0}^{k}=Q \Sigma R
$$

with $Q=\left[\begin{array}{llll}q_{1} & q_{2} & \cdots & q_{n}\end{array}\right]$ and $R=Q^{-1}=\left[\begin{array}{llll}r_{1}^{T} & r_{2}^{T} & \cdots & r_{n}^{T}\end{array}\right]^{T}$.

Hence, for $p$ sufficiently close to $p_{0}$ but $p>p_{0}$, the number of critical zeros entering the right-half plane (or vice versa) can be determined by the condition

$$
\cos \left(\frac{2 h \pi+\pi+\theta}{m}\right)>0(<0), \quad h=0, \cdots, m-1 .
$$




\section{PARAMETER-DEPENDENT POLYNOMials}

In the sequel, we will consider the following class of parameter-dependent polynomials:

$$
f(s, p):=P_{0}(s)+\sum_{k=1}^{n_{q}} P_{k}(s) p^{k},
$$

with the parameter $p \in \mathbb{R}$. In principle, there are several ways to compute the stability crossing set $\mathcal{P}$ by using the methodology presented in the previous sections. The standard method consists in finding a state-space representation that has the characteristic function given by $(24)^{1}$. Such a method was largely used in the context of robust analysis (see, e.g., [8], [1], [2] and the references therein). For the sake of brevity, such an approach will be omitted here.

In the sequel, we will propose however a different method which is inspired by the static output feedback control problem for SISO systems. Introduce now the following Hurwitz matrix associated to some polynomial

$$
A(s)=\sum_{i=0}^{n_{a}} a_{i} s^{n_{a}-i}
$$

and denoted by $H(A)$ :

$$
H(A):=\left[\begin{array}{ccccc}
a_{1} & a_{3} & a_{5} & \ldots & a_{2 n_{a}-1} \\
a_{0} & a_{2} & a_{4} & \ldots & a_{2 n_{a}-2} \\
0 & a_{1} & a_{3} & \ldots & a_{2 n_{a}-3} \\
0 & a_{0} & a_{2} & \ldots & a_{2 n_{a}-4} \\
\vdots & & & \ddots & \vdots \\
0 & 0 & 0 & \ldots & a_{n_{a}}
\end{array}\right] \in \mathbb{R}^{n_{a} \times n_{a}},
$$

where the coefficients $a_{l}=0$, for all $l>n_{a}$. Next, define the matrix pencil:

$$
\Lambda(\lambda):=\operatorname{det}(\lambda U+V),
$$

with $U, V$ given by:

$$
\begin{aligned}
U & =\left[\begin{array}{lllll}
I & & & \\
& \ddots & & \\
& & I & \\
& & H\left(P_{n_{q}}\right)
\end{array}\right] \\
V & =\left[\begin{array}{cccc}
0 & -I & \cdots & 0 \\
\vdots & \vdots & \ddots & \vdots \\
0 & 0 & \cdots & -I \\
H\left(P_{0}\right) & H\left(P_{1}\right) & \cdots & H\left(P_{n_{q}-1}\right)
\end{array}\right],
\end{aligned}
$$

where the identity, and the zero-blocks matrices have appropriate dimension, and $H\left(P_{i}\right) \in \mathbb{R}^{\left(n+n_{q}\right) \times\left(n+n_{q}\right)}$, for all $i=1,2, \ldots, n_{q}$ represents the corresponding Hurwitz matrix ${ }^{2}$ associated to the polynomial $P_{i}(s)$ defined above.

The following result gives the characterization of the characteristic roots of $f$ given by (24) on the imaginary

\footnotetext{
${ }^{1}$ by using, for example, appropriate companion forms and defining all the coefficients of $s^{k}$ as appropriate polynomials in the variable $p$, etc.

${ }^{2}$ Independently of the order of the polynomial $P_{i}\left(i=1, \ldots, n_{q}\right), H\left(P_{i}\right)$ will be constructed as a $\left(n+n_{q}\right) \times\left(n+n_{q}\right)$ matrix by setting the coefficients of high-order terms as zeroes, that is $q_{h}=0$, for all $h>\operatorname{deg}\left(P_{i}\right)$.
}

axis as a function of $T$, and represents a generalization of the method proposed by [3] in the context of static output feedback for SISO systems:

Proposition 5: Let $\lambda_{1}<\lambda_{2}<\ldots \lambda_{h}$, with $h \leq n+n_{q}$ be the real eigenvalues of the matrix pencil

$$
\Lambda(\lambda)=\operatorname{det}(\lambda U+V) .
$$

Then the parameter-dependent polynomial $f$ has some characteristic roots on the imaginary axis if and only if $T=\lambda_{i}$, $i=1,2, \ldots h$. Furthermore, if there are $r$ unstable roots $\left(0 \leq r \leq n+n_{d}\right)$ for $p=p^{*}, p^{*} \in\left(\lambda_{i}, \lambda_{i+1}\right)$, then, there are $r$ unstable roots for any parameter $p \in\left(\lambda_{i}, \lambda_{i+1}\right)$. The same property holds for the intervals $\left(0, \lambda_{1}\right)$ and $\left(\lambda_{h}, \infty\right)$.

In conclusion, as derived in the Proposition 5 above, the set of crossing parameters $\mathcal{P}$ is given by the set of generalized eigenvalue of the corresponding matrix pencil $\Lambda$. For each $p_{0} \in \mathcal{P}$ a simple test will give the set of corresponding characteristic roots on the imaginary axis. Let $j \omega_{0}$ one such a critical root. The remaining point to be clarified is the characterization of the crossing direction in some neighborhood of $p_{0}$. The crossing characterization derived in the previous section rewrites as follows in the simple and multiple root case respectively:

Proposition 6: Let $j \omega_{0}$ be a simple zero of $f(s, p)$ corresponding to the parameter $p=p_{0}$. If

$$
\operatorname{Re}\left\{\frac{\left.\frac{d f\left(j \omega_{0}, p\right)}{d p}\right|_{p=p_{0}}}{\left.\frac{d f\left(s, p_{0}\right)}{d s}\right|_{s=j \omega_{0}}}\right\}<0, \quad(>0)
$$

then for any $p$ sufficiently close to $p_{0}$ but $p>p_{0}, j \omega_{0}$ enters the right-half plane (or vice versa).

Finally, if

$$
\operatorname{Re}\left\{\frac{\left.\frac{d f\left(j \omega_{0}, p\right)}{d p}\right|_{p=p_{0}}}{\left.\frac{d f\left(s, p_{0}\right)}{d s}\right|_{s=j \omega_{0}}}\right\}=0,
$$

then for any $p$ sufficiently close to $p_{0}$ but $p>p_{0}, j \omega_{0}$ enters the right-half plane (or vice versa) if

$$
\operatorname{Re}\left\{\frac{\left.\frac{d^{2} f\left(j \omega_{0}, p\right)}{d p^{2}}\right|_{p=p_{0}}}{\left.\frac{d f\left(s, p_{0}\right)}{d s}\right|_{s=j \omega_{0}}}\right\}<0 \quad(>0) .
$$

Proposition 7: Let $j \omega_{0}$ be a repeated zero of $\left.f(s, p)\right)$ with multiplicity $m$, corresponding to the parameter $p=p_{0}$. If

$$
m ! \frac{\left.\frac{d f\left(j \omega_{0}, p\right)}{d p}\right|_{p=p_{0}}}{\left.\frac{d^{m} f\left(s, p_{0}\right)}{d s^{m}}\right|_{s=j \omega_{0}}}=0,
$$

then for any $p$ sufficiently close to $p_{0}$ but $p>p_{0}$, the zeros corresponding to $j \omega_{0}$ can be expanded by the Puiseux series

$$
\begin{aligned}
j \omega_{0} & +\left|m ! \frac{\left.\frac{d^{2} f\left(j \omega_{0}, p\right)}{d \tau^{2}}\right|_{p=p_{0}}}{\left.\frac{d^{m} f\left(s, p_{0}\right)}{d s^{m}}\right|_{s=j \omega_{0}}}\right|^{\frac{1}{m}} e^{j \frac{2 h \pi+\pi+\theta}{m}}\left(p-p_{0}\right)^{\frac{1}{m}} \\
& +\cdots, \quad h=0,1, \cdots, m-1,
\end{aligned}
$$

where $\theta \in[0,2 \pi]$ is the phase angle of

$$
\frac{\left.\frac{d^{2} f\left(j \omega_{0}, p\right)}{d p^{2}}\right|_{p=p_{0}}}{\left.\frac{d^{m} f\left(s, p_{0}\right)}{d s^{m}}\right|_{s=j \omega_{0}} .}
$$


Hence, for $p$ sufficiently close to $p_{0}$ but $p>p_{0}$, the number of critical zeros entering the right-half plane (or vice versa) can be determined by the condition

$$
\cos \left(\frac{2 h \pi+\pi+\theta}{m}\right)>0 \quad(<0), \quad h=0,1, \cdots, m-1 .
$$

\section{PSEUdO-DELAY AND PARAMETER-DEPENDENT POLYNOMIALS}

There exists several frequency-domain methods for handling the stability analysis of linear systems with commensurate delays (see, for instance, [7], [10] and the references therein). In the sequel, we shall focus on the pseudo-delay technique (see, e.g., [11], [12] for further details).

The characteristic function of a linear delay system:

$$
\dot{x}(t)=\sum_{i=0}^{n_{c}} x(t-i \tau),
$$

with $x \in \mathbb{R}^{n}$ is given by the quasipolynomial:

$$
f\left(s ; e^{-s \tau}\right):=P_{0}(s)+\sum_{i=1}^{n_{d}} P_{i}(s) e^{-i s \tau} .
$$

Roughly speaking, the idea of the approach is to use the bilinear transformation associating to $z=e^{-s \tau}$ the "quantity":

$$
z:=\frac{1-\lambda T}{1+\lambda T}, \quad T>0 .
$$

and the parameter-dependent polynomial:

$$
\begin{aligned}
\tilde{f}(s, T) & :=(1+s T)^{n} f\left(s, \frac{1-s T}{1+s T}\right) \\
& =\sum_{i=0}^{n_{d}} P_{i}(s)(1-T s)^{i}(1+T s)^{n_{d}-i} .
\end{aligned}
$$

It is easy to see that the imaginary value $s=j \omega_{0}$ with $\omega_{0}>0$ is a characteristic root of $f\left(s, e^{-s \tau}\right)$ for some $\tau_{0}>0$ iff $s=j \omega_{0}$ is also a root of $\tilde{f}\left(s, T_{0}\right)$ for some $T_{0}>0$, and the relation between $\tau_{0}$ and $T_{0}$ can be explicitly derived (see, e.g. [13], [12]).

Based on the theory presented in the paper, it is easy to see that the corresponding set of $T$ for which, at least, one characteristic root of $\tilde{f}$ lies on $\partial \mathbb{C}_{+}$will be given by computing the generalized eigenvalues of some appropriate matrix pencil associated to $\tilde{f}$. In other words, crossing detection for commensurate delays systems is reduced to finding parameters $T$ such that $\tilde{f}$ has characteristic roots on the imaginary axis. In conclusion, the generalized eigenvalues of $\Lambda$ constructed for $\tilde{f}$ will define the values of the parameter $T$ for which crossing exists. Next, for each $T_{0} \in\left\{\lambda_{1}, \ldots, \lambda_{h}\right\}$, we will compute the characteristic roots of the polynomial $\tilde{f}\left(s, T_{0}\right)$ on $\partial \mathbb{C}_{+}$. The set of frequencies corresponding to all these roots on the imaginary axis will define the frequency crossing set $\Omega$. Next, consider some frequency $\omega_{0} \in \Omega$, which corresponds to $T_{0}$. Then, the corresponding delay crossing set $\mathcal{T}_{\omega_{0}}$ will be derived by solving the equation:

$$
e^{-j \omega_{0} \tau}=\frac{1-j \omega_{0} T_{0}}{1+j \omega_{0} T_{0}}
$$

Finally, the crossing direction characterization follows the same procedures as in [4], [6], and it is omitted since it is out of the scope of the paper.

Remark 2: The detection of the crossing frequency set proposed here is quite distinct from the procedures proposed by [11], [12].

\section{CONCLUDING REMARKS}

This paper addressed a robust stability analysis of a class of linear systems depending on some real parameter (polynomial dependence). The approach considered here makes use of two ingredients: the computation of the generalized eigenvalues of an appropriate matrix pencil, combined with an operator perturbation methodology. A complete crossing characterization is proposed in the simple, semi-simple, and multiple, but not semi-simple characteristic root cases. Both state-representation and parameter-dependent polynomial cases have been presented. Some connections with some method encountered in the analysis of time-delay systems was also proposed.

\section{REFERENCES}

[1] Barmish, B.R.: New tools for robustness of linear systems (MacMillan, 1994).

[2] Bhattacharyya, S.P., Chapellat, H., and Keel, L.H.: Robust control: The parametric approach (Prentice Hall, 1995).

[3] Chen, J.: Static output feedback stabilization for SISO systems and related problems: solutions via generalized eigenvalues, in Control Theory and Advanced Tech. 10 (1995) 2233-2244.

[4] Chen, J., Fu, P. and Niculescu, S.-I.: Asymptotic behavior of imaginary zeros of linear systems with commensurate delays. Proc. IEEE Conf. Dec. Contr. (2006) San Diego, USA.

[5] Deif, A.S.: Advanced Matrix Theory for Scientists and Engineers (Abacus Press: UK, 1982).

[6] Fu, P., Chen, J. and Niculescu, S.-I.: High-order analysis of critical stability properties of linear time-delay systems. in Proc. 2007 American Contr. Conf., New York (2007) (to be presented).

[7] Gu, K., Kharitonov, V.L. and Chen, J.: Stability and robust stability of time-delay systems. (Birkhauser: Boston, 2003).

[8] Hinrichsen, D. and Pritchard, A. J.: Mathematical systems theory I. Modelling, state space analysis, stability and robustness (Springer Verlag: New York, TAM, vol. 48, 2005).

[9] Kato, T.: Perturbation Theory for Linear Operations (Springer-Verlag: Berlin, 1995)

[10] Niculescu, S.-I.: Delay effects on stability. A robust control approach (Springer-Verlag: Heidelberg, LNCIS, vol. 269, 2001).

[11] Olgac, N. and Sipahi, R.: An exact method for the stability analysis of time-delayed linear time-invariant (LTI) systems. IEEE Trans. Automat Contr., 47 (2002) 793-797.

[12] Sipahi, R.: Cluster treatment of characteristic roots, CTCR, A unique methodology for the complete stability robustness analysis of lineat time invariant multiple time delays against delay uncertainties (Ph.D. Thesis, University of Connecticut, Storrs, 2005).

[13] Thowsen, A.: An analytical stability test for a class of time-delay systems. IEEE Trans. Automat. Contr. AC-25 (1981) 735-736.

[14] Thowsen, A.: The Routh-Hurwitz method for stability test for a class of time-delay systems. Int. J. Contr. 33 (1981) 991-995. 